Service social

\title{
La réussite des interventions de groupe en contexte difficile. L'éclairage apporté par le Programme d'action communautaire pour les enfants (PACE)
}

\section{André Beaudoin}

Volume 46, numéro 2-3, 1997

Groupes - Symposium 1997

URI : https://id.erudit.org/iderudit/706760ar

DOI : https://doi.org/10.7202/706760ar

Aller au sommaire du numéro

Éditeur(s)

École de service social de l'Université Laval

ISSN

1708-1734 (numérique)

Découvrir la revue

Citer cet article

Beaudoin, A. (1997). La réussite des interventions de groupe en contexte difficile. L'éclairage apporté par le Programme d'action communautaire pour les enfants (PACE). Service social, 46(2-3), 65-76.

https://doi.org/10.7202/706760ar d'utilisation que vous pouvez consulter en ligne. 


\section{La réussite des interventions de groupe en contexte difficile L'éclairage apporté par le Programme d'action communautaire pour les enfants (PACE)}

André BEAUDOIN

Le texte qui suit fournit certaines réflexions sur l'intervention du service social des groupes à partir de certaines constatations faites dans l'évaluation du Programme d'action communautaire pour les enfants (PACE) au Québec. La présentation se situe dans le contexte des transformations et des bouleversements qui se produisent en cette fin de siècle, en tenant compte des possibilités nouvelles qui en résultent. Quelques résultats de l'évaluation des projets du PACE seront donnés dans le but d'en faire ressortir les implications pour le service social des groupes.

\section{LES TRANSFORMATIONS ET BOULEVERSEMENTS ACTUELS : POSSIBILITÉS NOUVELLES}

Le service social subit l'influence de la société dans laquelle il s'exerce à cause de l'interface où se trouvent la plupart de ses actions et les grands phénomènes de l'heure et à cause de son interdépendance avec les situations, les orientations et les tendances existant à un moment donné de l'histoire, que ce soit au niveau mondial, national ou local. La mondialisation des marchés et de l'économie met bien 
en évidence l'interdépendance entre les différents pays. Des organismes comme la Banque mondiale et le Fonds monétaire international sont là pour rappeler à différents endroits qu'aucun gouvernement ne peut aujourd'hui se permettre de maintenir des taux d'endettement élevés. Plusieurs pays, la plupart du temps parmi les plus pauvres, se le font rappeler durement et leurs populations en subissent les contrecoups inévitables.

Les orientations de la politique sociale ne peuvent plus se définir et encore moins se réaliser en vase clos. Il y a une interdépendance de plus en plus étroite entre l'économie internationale et les types de politique sociale que peuvent se donner les différents pays. À titre d'exemple, certaines mesures, comme celles de l'assurance santé, mises en place à l'intérieur de l'État-providence et qui ont pour caractéristique principale de rendre gratuits et universels les services de santé, sont remises en cause et menacées si le même type de programme ne se retrouve pas dans un pays voisin avec lequel il y a libre-échange sur le plan économique. Les marges de manœuvre des gouvernements deviennent plus faibles et la tendance sera de se rapprocher du système voisin, surtout s'il s'agit de celui d'un pays plus fort, en raison des déséquilibres présumés que cela occasionne dans les forces du marché.

II se fait aussi à l'intérieur des différents pays une remise en question de plus en plus forte des formes trop centralisées d'organisation de services à cause de leur forte rigidité et de leur trop grande bureaucratie. Celles-ci débouchent sur des services déshumanisés, laissant peu de place au respect des personnes et de leurs différences; de plus, elles entravent la spontanéité ou la créativité souvent nécessaires dans plusieurs situations. II reste sans doute des auteurs qui continuent de soutenir et de défendre l'État-providence (Leonard, 1990), mais il s'en trouve aussi d'autres pour soutenir plutôt un engagement minimal de l'État dans les programmes de bien-être public (Wineman, 1984).

II apparaît de plus en plus clair que nous entrons dans le $21^{\mathrm{e}}$ siècle en pensant que plusieurs des modèles de politique sociale centrée sur l'État-providence ont vécu et qu'ils sont en voie de remplacement. En même temps, on insiste de plus en plus sur le fait que l'orientation à prendre pour corriger les déficiences des grandes institutions bureaucratiques de bien-être public est leur remplacement 
par des organisations définies sur une échelle plus humaine. Espérons malgré tout qu'on ne jettera pas le bébé avec l'eau du bain! Et qu'on réussira, dans le nouveau contexte, à conserver les acquis antérieurs. Dans les changements en cours, il faut accorder une attention prioritaire aux besoins humains des populations les plus pauvres, vulnérables et victimes d'exclusion. II faut faire disparaître le plus possible les obstacles à leur intégration et faire en sorte qu'elles puissent prendre en main leur destinée et s'approprier le plus possible les moyens nécessaires à leur insertion sociale (Moreau et Leonard, 1989).

C'est dans cette perspective qu'il faut situer l'insistance mise sur l'empowerment des populations, c'est-à-dire l'appropriation par les différents groupes de base de l'exercice du pouvoir. Une telle orientation dépasse les clivages qu'on retrouve dans certaines conceptions de l'empowerment. L'accent n'est pas mis uniquement sur les seules caractéristiques individuelles vues comme un instrument à l'appropriation d'un plus grand pouvoir des personnes sur leur destinée. Mais l'empowerment est en même temps vu comme un processus de développement d'un plus grand pouvoir des groupes en tant qu'outil de changement social (Adams, 1990; Lee, 1994; Le Bossé, 1995; Zimmerman, Bonner et Kovach, 1996).

L'orientation privilégiée ici tient compte à la fois de la place qu'occupe la personne dans son environnement et de l'importance accordée à cet environnement comme facteur d'influence sur les personnes. Ce sont là deux aspects qui sont - est-il utile de le rappeler? - essentiels à la pratique du service social. C'est dans ce contexte qu'il est possible d'analyser certaines formes d'action collective auprès des enfants et de leur famille, comme celle du Programme d'action communautaire pour les enfants (PACE) à l'évaluation duquel nous avons participé au cours des dernières années.

\section{LES POSSIBILITÉS DU PACE DANS UN CONTEXTE D'EMPOWERMENT}

Le PACE a été mis sur pied par le gouvernement du Canada pour faire suite à la Conférence de Rio sur l'enfance. II fait partie d'un ensemble d'actions dont le but explicite est de soutenir les enfants surtout les jeunes enfants - et leur famille, qui appartiennent aux populations les plus vulnérables. Plus précisément, le PACE vise à 
réduire les obstacles auxquels sont confrontés les jeunes enfants des milieux vulnérables et leurs parents, que ce soit sur le plan de leur santé, de leur vie sociale, de leur environnement ou de leur développement. L'ensemble des principes directeurs qui servent de guide au PACE (Gouvernement du Canada, 1992a et 1992b) sont les suivants :

- Les enfants d'abord : le bien-être de l'enfant est la première considération du programme.

- Le renforcement et le soutien aux familles : il faut aider les parents à prendre soin de leurs enfants.

- L'équité ou l'accessibilité : chaque enfant doit avoir les mêmes chances que les autres de développer son potentiel, quel que soit le groupe culturel auquel il appartient.

- Le partenariat entre les organisations concernées : ce partenariat est essentiel à la mise en place, sur une base continue, des moyens appropriés.

- L'action communautaire : on reconnaît par cette action que les parents et la communauté ont un rôle essentiel à jouer dans ces programmes.

- La souplesse d'adaptation : il faut faire preuve de souplesse en tenant compte des besoins des différentes communautés.

Plus spécifiquement au Québec, à la suite d'un accord entre le gouvernement du Québec et celui du Canada (ministère de la Santé et des Services sociaux - Santé Canada, 1993), le PACE se réalise selon trois orientations principales :

1. Promouvoir le développement des compétences parentales, l'adaptation des parents à leur rôle, la valorisation du rôle du père ainsi que la qualité de la relation parent-enfant.

2. Promouvoir la sécurité, la croissance et le développement optimal de l'enfant sur les plans physique, affectif, social et cognitif.

3. Offrir aux enfants et aux parents particulièrement vulnérables le soutien dont ils ont besoin pour prévenir les problèmes d'adaptation.

Le PACE fournit un financement à des projets issus du milieu et réalisés à l'intérieur des communautés pour la promotion de la santé 
et du bien-être des enfants, pour la prévention, pour l'intervention précoce et intensive dans les familles à risque et pour la mise en œuvre d'approches du milieu auprès des enfants et des familles.

Dans l'ensemble, les principales caractéristiques des projets du PACE au Québec tels qu'ils se dégagent de l'étude que nous avons effectuée (Beaudoin, Camiré, Germain, Champoux et Turcotte, 1997) sont les suivantes:

1. Les projets du PACE sont proportionnellement plus nombreux au Québec que dans les autres régions du Canada. En 1996, il y avait 182 projets réalisés sur une base récurrente au Québec pour un budget total d'environ 35 millions de dollars.

2. La presque totalité des territoires de centres locaux de services communautaires (CLSC) où la vulnérabilité des jeunes et de leurs familles est élevée sont touchés par un projet ou l'autre du PACE.

3. Les trois quarts des projets se situent dans des organismes communautaires et le quart dans des établissements du réseau public de la santé et des services sociaux, surtout des CLSC.

4. Les organismes communautaires dans lesquels se réalisent les projets sont de très petite taille : plus des trois quarts ont moins de six employés permanents à plein temps. Les établissements publics où l'on retrouve des projets sont quant à eux de taille beaucoup plus grande : ils comptent tous plus de 50 employés.

5. Les organismes communautaires responsables des projets sont dans une forte proportion des organismes de services à l'enfance et à la famille (73 \%); il peut cependant s'agir aussi d'autres organismes, comme ceux qui s'adressent à des femmes, surtout aux femmes violentées, et à leurs enfants.

6. Pour ce qui est de la réalisation de leur action sur le terrain, les projets font appel dans une large mesure au bénévolat: plus de $57 \%$ des organismes comptent 30 bénévoles et plus.

7. Les contributions annuelles moyennes versées par Santé Canada aux projets sont de l'ordre de 46600 \$. La plus grande partie des organismes reçoivent des contributions annuelles qui se situent entre $30000 \$$ et $60000 \$$. 
8. Pour les organismes communautaires, la contribution financière reçue du PACE est essentielle à leurs activités auprès des enfants, de leur famille et du milieu : elle est plus grande dans plus de la moitié des cas au tiers du budget total de ces organismes.

Même si ces quelques caractéristiques sont essentielles pour situer le contexte de réalisation de l'action des projets du PACE au Québec, c'est plutôt à l'examen du contenu de l'intervention que nous nous attarderons ici. De l'examen de l'ensemble des activités accomplies dans les projets, il ressort trois axes principaux d'intervention. Le premier est l'accompagnement fourni aux parents dans l'exercice de leurs tâches parentales: on le retrouve présent dans $86 \%$ des projets. Ainsi, environ neuf projets sur dix ont pour but de fournir du soutien direct aux parents dans l'apprentissage de nouveaux modes d'interaction avec leurs jeunes enfants et de favoriser l'acquisition d'habiletés éducatives. Le deuxième axe est la stimulation de l'enfant d'âge préscolaire : cette forme d'intervention se retrouve dans $57 \%$ des projets. Ainsi, les projets comportent, dans une proportion importante, en même temps des activités de stimulation réalisées auprès des enfants de 2 à 5 ans. Le but poursuivi par ces activités est d'améliorer le fonctionnement des jeunes enfants avant leur entrée à l'école au moyen de la stimulation sur les plans psychomoteur, social, affectif et cognitif. En troisième lieu, dans $53 \%$ des projets, le soutien aux parents de jeunes enfants occupe une place importante. Les activités de soutien sont fournies aux parents dans le but de les appuyer et de les soutenir dans l'exercice de leurs responsabilités parentales et d'alléger à certains moments cruciaux le fardeau de leurs tâches.

À partir de l'examen plus poussé des formes que prennent les activités dans les projets, on se rend compte que ce sont les activités de groupe qui constituent le moteur principal des interventions dans un nombre considérable de projets au Québec. En effet, l'analyse des données recueillies montre que cette forme d'activités est majoritairement privilégiée pour la réalisation des objectifs d'intervention dans $77,8 \%$ des projets, alors que l'intervention individuelle et l'aide matérielle constituent les autres formes d'activités utilisées. Les groupes mis en place prennent tantôt la forme de groupes fermés (dans $47,8 \%$ des activités), tantôt celle de groupes ouverts (dans $30 \%$ des cas). Les activités réalisées en groupes fermés le sont au moyen d'ateliers hebdomadaires plus ou moins dirigés à 
l'intérieur desquels des intervenants ou animateurs abordent des contenus qui s'enchaînent d'une rencontre à l'autre. On utilise aussi quelquefois des programmes d'activités qui se donnent selon un modèle prédéfini, par exemple le programme "Y'a personne de parfait " (YAPP). Ces rencontres organisées sous la forme de groupes fermés requièrent la participation des parents pendant plusieurs semaines, la plupart du temps entre 8 et 12 semaines.

Quant aux activités qui se donnent sous la forme de groupes ouverts, elles n'exigent pas une présence aussi régulière de la part des parents. Les rencontres sont souvent aussi structurées par thèmes, mais ces derniers sont indépendants les uns des autres, ce qui permet aux participants de joindre les groupes en fonction de leurs besoins particuliers.

L'examen plus poussé de l'intervention effectuée en groupe auprès des mères fait ressortir une grande diversité dans la nature des activités, comme l'ont souligné Turcotte et ses collaborateurs (Turcotte, Samson, Lessard et Beaudoin, 1997, p. 79-86). Le graphique qui suit montre de manière explicite comment les modalités de l'intervention de groupe se font dans les projets en tenant compte de leurs objectifs et de la structuration des groupes.

\section{FIGURE 1}

Modalités d'intervention de groupe auprès des parents

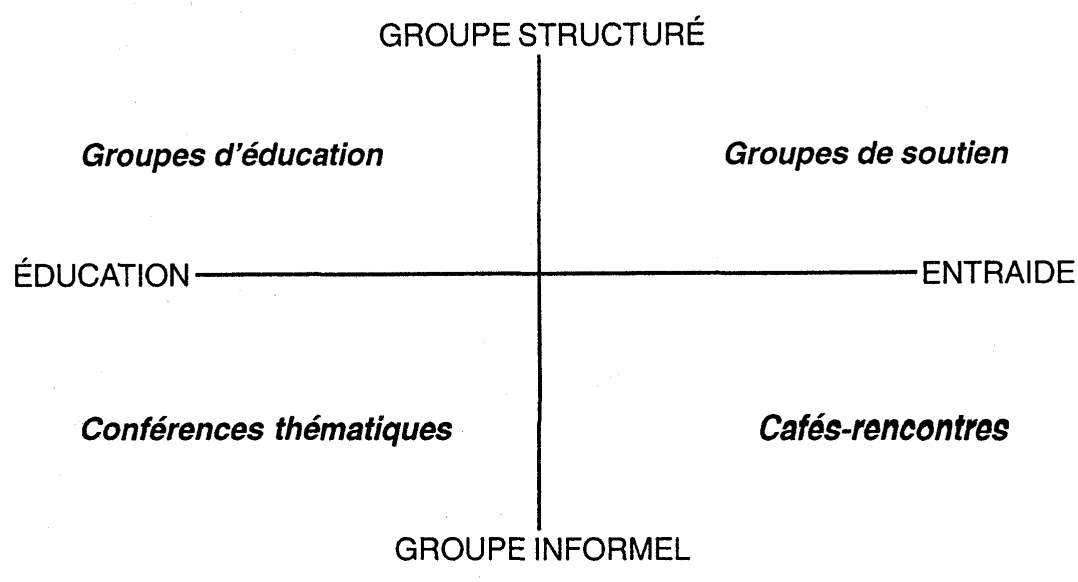


En ce qui concerne le contenu des objectifs (axe horizontal), certains projets ont des préoccupations d'éducation ou, autrement dit, de transmission de connaissances : ils visent à enrichir les connaissances des parents, surtout celles des mères, sur des thèmes comme le développement de l'enfant, la stimulation et la communication entre parents et enfants. D'autres projets visent plutôt à encourager l'entraide : ils partent davantage des problèmes soulevés par les mères et tentent d'y apporter des solutions en mettant à contribution les compétences des participantes. Sur l'axe vertical, on voit que les projets procèdent tantôt selon une approche informelle en misant sur la spontanéité à partir des préoccupations du moment, tantôt d'une manière plus structurée en encadrant les réunions à l'aide d'un ensemble d'activités organisées sous la forme d'un programme défini à l'avance à durée déterminée s'adressant à un groupe fermé. II est ainsi possible de regrouper autour de quatre modes principaux d'intervention de groupe les activités des projets du PACE :

1. Des groupes d'éducation : ils se centrent sur l'information et les outils à transmettre aux mères participantes et ils se réalisent la plupart du temps dans le cadre de groupes « fermés ". L'intervenant est très fréquemment l'animateur dans ces groupes et le contenu est structuré pour faciliter l'apprentissage autour de sujets relatifs au rôle parental.

2. Des groupes de soutien : l'objectif premier de ces groupes est de créer un cadre qui permet l'entraide entre les mères dans l'exercice de leur rôle parental. Ces groupes sont la plupart du temps animés par les intervenants et quelquefois par des bénévoles. On y part de la réalité vécue quotidiennement par les mères en les mettant à contribution. Dans les groupes de soutien, il s'établit une continuité entre les activités de groupe et le milieu naturel.

3. Des conférences thématiques: sous cette forme, les groupes sont d'habitude ouverts. Même si les deux parents sont invités à participer, c'est surtout les mères qui sont rejointes. Les conférences sont la plupart du temps accompagnées de discussion et d'échanges entre les participants, les invités et les animateurs. Ces rencontres sont l'occasion d'aborder avec les participants des thèmes clés qui touchent le développement de l'enfant et l'exercice du rôle parental. 
4. Des cafés-rencontres : cette dernière forme d'activités, très informelle, consiste à choisir des endroits et des moments précis où les mères peuvent discuter dans un cadre informel entre elles ou avec des intervenantes. Les mères s'y rendent selon leur disponibilité. II n'y a pas nécessairement de programme déterminé, mais plutôt une forme d'animation spontanée et informelle à partir des préoccupations du moment.

\section{IMPLICATIONS POUR L'INTERVENTION DU SERVICE SOCIAL DES GROUPES}

Ces quelques constatations ont permis de montrer comment, par l'intermédiaire des projets du PACE, l'intervention de groupe devient une nouvelle possibilité d'action en milieu naturel avec des parents et des jeunes enfants. Même si tous les projets ne sont pas au même degré d'avancement à cet égard, la forme d'intervention prédominante dans une quantité importante de projets se rapproche des principes du service social des groupes. En plus du soutien que les activités procurent aux mères participantes, c'est souvent l'occasion pour elles de s'impliquer, de prendre une part active aux groupes, de contribuer au recrutement et même quelquefois d'organiser des activités et de faire l'animation des rencontres. Elles y vivent ainsi un sentiment de contribution et se sentent moins isolées et moins dépendantes socialement, dans une perspective d'appropriation et d'empowerment face à leur situation (Berman-Rossi, 1992). Dans ces groupes, les intervenants exercent un rôle de catalyseurs. Ils favorisent l'accès aux ressources. Ils garantissent la qualité de l'accueil et du contenu des activités. Ils sont enfin là pour fournir le contexte nécessaire à la participation des parents, surtout des mères, à des activités de socialisation à l'exercice de leurs rôles parentaux. En même temps, les activités de groupe contribuent à la diminution de l'isolement social des mères, à l'augmentation de leur sens du partage et à l'amélioration de leur sentiment d'être en meilleur contrôle de leur propre situation.

Si l'on généralise en fonction du service social des groupes, les constatations précédentes mettent clairement en évidence qu'il y a de la place pour l'insertion de l'intervention de groupe dans la communauté par l'intermédiaire d'actions comme celles qu'on retrouve 
dans le PACE. Ces dernières se réalisent directement dans le milieu et prennent la forme d'initiatives au profit du milieu. L'intervention de groupe s'y introduit comme une composante qui fait partie du processus même de l'action communautaire entreprise dans le milieu. Elle s'y insère d'une manière plus naturelle et plus ouverte que lorsqu'elle se réalise dans un milieu institutionnel et fermé. Elle n'y est pas toujours introduite comme une activité spécifique structurée externe qui s'adresse à un groupe défini à l'avance, mais elle fait partie de l'action même des projets entrepris à l'égard des enfants et de leur famille en situation de vulnérabilité. C'est là une implication importante pour le travail en groupe.

Malgré ces avantages évidents, l'utilisation des méthodes de groupe, telle qu'elle se fait dans les projets PACE, suscite un certain nombre de dilemmes et de défis qu'il ne faut surtout pas ignorer et qui ne sont pas étrangers à la pratique du service social des groupes, comme l'ont souligné récemment certains auteurs (Schopler et Galinsky, 1995). Ces dilemmes doivent faire l'objet d'un examen attentif, même si nous ne faisons que les énumérer ici. II y a en premier lieu la question de l'équilibre à maintenir entre les différents niveaux de structuration des activités dans les groupes où se retrouvent les mères et les enfants. Certains prétendent que les activités moins formelles rejoignent plus facilement les membres des familles plus vulnérables, alors que d'autres jugent que des activités plus structurées à caractère éducatif sont essentielles et incontournables, surtout lorsqu'on vise les populations vulnérables comme c'est la plupart du temps le cas ici.

Un deuxième dilemme concerne la difficulté de recrutement des membres. Pour mettre en branle un processus de groupe dans un projet, il faut une masse critique de membres. Dans les faits, certains projets ont eu de la difficulté à attirer un minimum de membres, surtout lorsqu'on essaie de recruter ceux-ci en fonction de critères de vulnérabilité. C'est en particulier le cas dans les milieux ruraux et plus isolés quand les mères doivent parcourir des distances importantes pour participer aux réunions et qu'elles n'ont pas accès au transport.

Un dernier dilemme vient de la difficulté à maintenir une certaine cohésion entre des participantes souvent très différentes les unes des autres surtout dans les groupes ouverts ou dans les groupes 
d'entraide, en particulier en milieu urbain où l'on trouve des gens de différents groupes ethniques et culturels. Le fait aussi que certaines mères aient un engagement plus fort comme membres du groupe, qu'elles soient plus assidues aux rencontres et aient une plus longue durée d'appartenance peut constituer un handicap pour l'insertion de nouveaux membres. L'intervenant est souvent confronté à la difficulté de dégager un terrain commun d'échange et d'entraide entre les mères. Si les participantes ne possèdent pas à un degré suffisant les habiletés sociales nécessaires à la création de relations adéquates, on se heurte à des difficultés supplémentaires.

En dépit de tout cela, il se dégage, des constatations de l'évaluation des projets du PACE au Québec, que sous plusieurs angles l'action réalisée s'avère un terrain fertile pour les activités du service social de groupe. Cette évaluation démontre l'existence de nouveaux contextes dans lesquels il est possible d'insérer des activités de l'intervention menée en service social des groupes à l'intérieur des milieux naturels de vie des enfants.

André BEAUDOIN

Professeur École de service social Université Laval

\section{Références bibliographiques}

ADAMS, R. (1990). Self-help, Social Work and Empowerment, Londres, Practical social Work; Macmillan Education.

Beaudoin, A., L. Camiré, A. Germain, L. Champoux et D. Turcotte (1997). L'action des projets sur le terrain, vol. 1 de L'évaluation des projets du PACE au Québec, Québec, Université Laval, Centre de recherche sur les services communautaires.

BERMAN-ROSSI, T. (1992). « Empowering through understanding stages of group development ", Social Work With Groups, vol. 15, nos 2-3, p. 239-255.

GOUVERNEMENT DU CANADA (1992a). Grandir ensemble : plan d'action canadien pour les enfants, Ottawa, Gouvernement du Canada.

GOUVERNEMENT DU CANADA (1992b). Grandir ensemble, programme d'action communautaire pour les enfants (PACE), Concept du programme, Ottawa, Santé Canada (document de discussion non publié). 
LE BosSÉ, Y. (1995). Étude exploratoire du phénomène de l'empowerment, Université Laval, Faculté des études supérieures, Thèse (Ph. D.) en psychologie.

LEE, J.A.B. ( 1994). The empowerment approach to social work practice, New York, Columbia University Press.

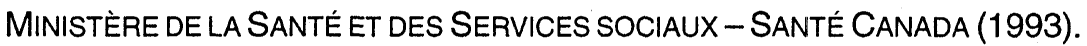
Protocole d'entente entre le gouvernement du Canada et le gouvernement du Québec concernant le programme d'action communautaire pour les enfants (PACE), Gouvernement du Québec et Gouvernement du Canada (document non publié).

MOREAU, M. et L. LEONARD (1989). Empowerment through a structural approach to social work : a report from practice, Montréal, École de service social.

Sen, G., A. GERMAIN, L.C. CHEN (dir.) (1994). Population policies reconsidered: health, empowerment, and rights, Boston, Harvard Center for Population and Development Studies, Harvard University Press.

LEONARD, P. (1990). "Contesting the welfare state in a neo-conservative era ", Journal of Progressive Human Services, 1, p. 11-25

SCHOPLER, J.H. et M.J. GALINSKY (1995). " Group Practice Overview », dans R.L. Edwards, Encyclopedia of Social Work, $19^{\mathrm{e}}$ édition, Washington, National Association of Social Workers, p. 1129-1144.

Turcotte, D., C. SAmson, G. Lessard et A. Beaudoin (1997). De l'intention à l'évaluation : la dynamique de réalisation des projets d'action communautaire, vol. 2 de L'évaluation des projets du PACE au Québec, Québec, Université Laval, Centre de recherche sur les services communautaires.

WINEMAN, S. (1984). The Politics of Human Services : Radical Alternatives to the Welfare State, Montréal, Black Rose Books.

ZIMMERMAN, B.J., S. BONNER et R. KOVACH (1996). Developing self-regulated learners : beyond achievement to self-efficacy, Washington, DC, American Psychological Association. 